\title{
Obesity: a challenge for science and society
}

\author{
Valentin Fuster
}

In 1995, 200 million adults were obese (BMI $>30 \mathrm{~kg} / \mathrm{m}^{2}$ ), and that number had reached 300 million by 2004. Obesity and overweight prevalence is rising in young people worldwide. Today, one in every ten children is overweight, totaling 155 million, 30-45 million of whom are classified as obese. This problem affects not only industrialized nations but also low-income and middle-income countries.

The risk of heart disease and stroke increases with increasing BMI. Additional associations have been made with abdominal obesity, insulin resistance and diabetes, raised blood pressure and lipid abnormalities (high triglycerides, low HDL cholesterol), which form the so-called metabolic syndrome. Patients who have the metabolic syndrome have a 1.5-3.0-fold increase in the risk of coronary disease and stroke. In the US, $4.2 \%$ of people aged 12-19 years are thought to be affected by the metabolic syndrome, and that percentage is rising. Thus, focus has shifted to school-age children.

Obesity appears to be a critical contributor to the development of the metabolic syndrome. Dynamic processes throughout life determine the development of obesity, and may occur at any time from before conception, through the embryonic and fetal stages, infancy, childhood and adolescence, and into adult life. These 'insults' are particularly critical in the early stages of life and can include any of at least three determinants: environment, lifestyle and psychologic factors (e.g. type and portion size of food, physical activity, attitude); biologic factors (i.e. metabolic adjustments); and genetic factors (i.e. familial transmission). The approach to treatment requires the identification and evaluation of the determinants particular to each individual.

Understanding the cellular and molecular mechanisms underlying the disorders of the metabolic syndrome and their links with each
In the US, $4.2 \%$ of people aged 12-19 years are thought to be affected by the metabolic syndrome, and that percentage is rising

$V$ Fuster is the Editorin-Chief of Nature Clinical Practice Cardiovascular Medicine.

\section{Competing interests}

The author declared he has no competing interests.

www.nature.com/clinicalpractice doi:10.1038/ncpcardio0070 other has been challenging but is enabling new treatments to be developed. The adipocyte, or fat cell of the abdominal adipose tissue, is thought to be a source of tumor necrosis factor and of other high cytokine concentrations (interleukins 6 and 18 and P-selectin). Tumor necrosis factor might cause impairment in insulin signaling leading to insulin resistance and hyperglycemia, both of which contribute to endothelial damage and atherogenesis. The other cytokine excesses may lead, directly or mediated by the liver, to an inflammatory response (C-reactive protein, fibrinogen), dyslipidemia (triglycerides, HDL cholesterol) and hypertension, all abnormalities of the metabolic syndrome that can independently contribute to the atherothrombotic process. Furthermore, cytokine excess might perpetuate obesity by interfering with inhibitors of food intake (leptins) acting at the site of the novel cannabinoid type-1 receptor of the hypothalamus.

Unfortunately, therapy for such a raised risk factor profile or the metabolic syndrome in the global population might not be affordable. Obesity must be prevented, thus preventing the development of the additional risk factor profiles, including diabetes. Given all that we know about obesity, its health consequences, and the need for community support, we must create healthier environments for our children. To do so will take strong commitment from the corporate world, national and local governments, the media, nongovernmental organizations and health-care providers. Creative public-private partnerships are urgently needed. According to the WHO, $60 \%$ of the worlds' children today are insufficiently physically active and consume too many calories for the inactive lives they lead. Our children and our grandchildren can still 'grow out of' the problem of obesity and overweight, if and only if all stakeholders accept their responsibility to change. 